\title{
Meta-analysis: Conceptual bases, statistical analysis and interpretation
}

\section{Metaanálisis: bases conceptuales, análisis e interpretación estadística}

\author{
Christian Fau* and Solange Nabzo
}

Fundación Oftalmológica 2020, Iberoamerican Cochrane Network, Santiago, Chile

\begin{abstract}
The term meta-analysis was first used by G.V. Glass in 1976 in an article called "Primary, secondary and meta-analysis of investigations" "Primaria, secundaria y meta-análisis de la investigación"). He used this term to refer to the statistical analysis of all the results obtained in different clinical studies regarding the same subject and that were to be analyzed together. At the beginning, this type of analysis was mainly used for the examination of social studies and psychology investigations, but later, during the 1980s, it became a popular method used in medicine; particularly in the cardiovascular, cancer and perinatal specialties. Nowadays it is not rare to find several medical articles using this method. For this study we decided to perform a meta-analysis and also combine the results of the studies because when the sample size increases, the statistical potential increases as well. Furthermore, when including studies and researches performed in different Health Centers, the results obtained can be easily generalized. Nevertheless, the meta-analysis method has its controversies, many of them due to an excessive use of the method together with a lack of methodological rigor; there are many limitations to be considered when evaluating the results of a meta-analysis. In this article we will focus mainly on the statistical aspects of the subject, from the point of view of the description of the methods, indications and interpretations, without specifying other details such as the protocols for carrying out a systematic review or statistical formulas. The focus will be on the analytical methods used in meta-analyses of controlled clinical trials evaluating therapeutic efficacy or adverse reactions.
\end{abstract}

Key words: Systematic reviews. Meta-analysis. EBM. Heterogeneity. Publication bias. Evidence-based medicine. Literature search. Sensitivity analyses. Subgroup analysis.

\section{Resumen}

El término metaanálisis fue empleado por primera vez por G.V. Glass en 1976, en un artículo titulado Primaria, secundaria y metaanálisis de la investigación, para referirse al análisis estadístico del conjunto de resultados obtenidos en diferentes ensayos clínicos sobre una misma cuestión, con la finalidad de evaluarlos de manera conjunta. Este tipo de investigación fue inicialmente aplicado en las ciencias sociales y psicología, y en la década de 1980 el metaanálisis se hizo cada vez más popular en medicina, particularmente en los campos de enfermedad cardiovascular, oncología y perinatal. En la actualidad son muy frecuentes los artículos que describen resultados de metaanálisis en publicaciones médicas. Una de las

Correspondence:

${ }^{*}$ Christian Fau

Avda. Presidente Riesgo, 5157

Dep. 212, Las Condes, Santiago, Metropolitana, Chile

E-mail: chfauf@gmail.com

2604-1731/@ 2020 Sociedad Mexicana de Oftalmología. Published by Permanyer. This is an open access article under the CC BY-NC-ND license (http://creativecommons.org/licenses/by-nc-nd/4.0/).
Date of reception: 20-04-2020

Date of acceptance: 12-06-2020

Available online: $09-11-2020$

Rev Mex Oftalmol (Eng). 2020;94(6):236-248

www.rmo.com.mx 
razones para realizar un metaanálisis y combinar los resultados de los estudios es que al aumentar el tamaño de la muestra aumenta su potencia estadística, además, incorporar trabajos efectuados en centros y lugares diferentes conlleva que los resultados obtenidos pueden ser más fácilmente generalizables. Los metaanálisis no han estado ni están libres de controversia, entre otras cosas porque, quizás, como suele ser habitual con todo lo que se pone de moda, ha sido utilizado con una frecuencia excesiva sin suficiente rigor metodológico, ya que existen múltiples limitaciones a tener en cuenta a la hora de evaluar los resultados de un metaanálisis. En este artículo nos vamos a centrar fundamentalmente en los aspectos estadísticos del tema, desde el punto de vista de la descripción de los métodos, indicaciones e interpretaciones, sin entrar en otros detalles, como pueden ser los protocolos de realización de una revisión sistemática o fórmulas estadísticas. El enfoque va a ser en los métodos de análisis utilizados en metaanálisis de ensayos clínicos controlados que evalúan eficacia terapéutica o reacciones adversas.

Palabras clave: Revisión sistemática. Metaanálisis. MBE. Heterogeneidad. Sesgo de publicación. Medicina basada en la evidencia. Búsqueda de literatura. Análisis de sensibilidad. Análisis de subgrupos.

\section{Introduction}

The first text that combined the evidence from different sources was made in 1861 by the English astronomer George Biddell Airy. In the medical area, the English statistician Karl Pearson, in 1904, grouped the statistics of medical and military facilities of South Africa and India, and concluded that the vaccine against intestinal fever was not effective, but it was not until 1976 that the term meta-analysis was employed for the first time by the psychologist G.V. Glass, in an article entitled Primary, Secondary, and Meta-analysis research. He used this term to refer to the statistical analysis of the set of results obtained from different clinical trials in order to evaluate them together. This type of research was initially used in the social sciences and psychology, and, in the 1980s, meta-analysis became increasingly popular in medicine, developing in 1989 the term meta-analysis, to index these studies in the Medical Subject Headings (MeSH) of the Index Medicus.

In the past, one of the main problems in medicine was the scarce research, also with an insufficient number of patients, published in a limited number of hardto-access printed journals. There was, then, a lack of information. So the initial reason for conducting a meta-analysis was to group the results of the few existing studies, thereby increasing the sample size and its statistical power. Today, with the development of digital systems and the volume of biomedical information, which is expanding exponentially worldwide, in many areas it has become impossible to read, critically assess and synthesize the current state of knowledge, and much less to keep an update on a regular basis. Currently, the problem is no longer a lack of information, but an excess of it. Dr. Sackett, father of evidence-based medicine, in a conference in December
1997 stated: «A doctor who wants to diagnose and treat his patient with what has truly proven its worth, needs to read 19 scientific articles every day of the year; something that is clearly a utopia». Therefore, review articles have become essential tools to keep up to date. However, initially, this was supplied by narrative reviews. Mulrow in 1987, and Oxman and Guyatten in 1988, attracted worldwide attention regarding the poor quality of these, many of them even with a clear intention of inducing specific behaviors, directed by pharmaceutical companies and other market players. Consequently, systematic reviews and meta-analyses arose as a way to systematically structure the information, in order to reduce biases. But meta-analyses have not been and will not be free of controversy, among other things because, perhaps, as is usual with everything that becomes fashionable, they have been used excessively without enough methodological rigor. This is a contradiction to what is precisely their purpose. Meta-analyses have multiple limitations to consider when evaluating their results; also, there are those who revere the numerical results without critically analyzing the procedures with which they were obtained. Therefore, today, one must always keep in mind the mnemonics GIGO (garbage-in, garbage-out) when evaluating the information of a meta-analysis ${ }^{1-6}$.

In this article we will focus mainly on the statistical aspects of the topic, from the point of view of the description of the methods, indications and interpretations, without discussing other details, such as the protocols for conducting a systematic review or statistic formulas which, although of great importance, have been previously analyzed in several publications. To deepen the protocolization of a systematic review, it is suggested to consult the Cochrane manual of systematic reviews and the PRISMA statement ${ }^{7-10}$; both articles are freely accessible on the Internet. The focus of 
this article will be on the methods of analysis used in a meta-analysis of controlled clinical trials that evaluate efficacy or adverse reactions (therapeutic). The more complex methods used for a meta-analysis of diagnostic tests, survival analysis or network meta-analysis ${ }^{11}$ will not be analyzed in this article, so the objective of this study is to analyze the different statistical and graphic methods that can be used when conducting a therapeutic meta-analysis, to allow a better understanding and interpretation of the results. To facilitate understanding, the examples will be made using data from the Cochrane Patching for corneal abrasion (Review) ${ }^{12}$ publication, that corresponds to a meta-analysis of 11 randomized controlled clinical trials evaluating the effect of an eye patch to repair or heal superficial corneal erosions smaller than $10 \mathrm{~mm}^{2}$. From this analysis, we only took the data about corneal repair rates with or without patch on the first day of follow-up.

\section{What is a meta-analysis?}

A systematic review is defined as a type of secondary research, research on what was investigated, prepared with a systematic approach to minimize biases and random errors, in which the unit of analysis are the original primary studies and not the patients. A systematic review, which is qualitative, may or may not include a meta-analysis, which is a quantitative analysis of the results of different independent studies with a similar design, which generally aims to produce a weighted estimate of the effect, using statistical methods that allow grouping the results of two or more studies. This also increases the precision for effect estimation. When the same problem is analyzed in different studies, it is foreseeable, by pure chance, to find differences between the results, which can be maximized in the case of small studies, since the estimation is less precise. The objective of using a meta-analysis to combine the results of the studies is that, by increasing the sample size, its statistical power increases. In addition, incorporating studies performed in different centers and places means that the results obtained can be more easily generalized. By obtaining a larger sample we reduce the probability of making a type II error. This error is the probability of not detecting a difference of a certain magnitude between the groups when it does exist or, what is the same, the probability of concluding in our study that there are no statistically significant differences when in fact there are. Even when there are effects of moderate or small magnitude but clinically important, these might go unnoticed in primary studies and be more clearly appreciated on a weighted measure.

Meta-analyses constitute an essential tool to synthesize the available scientific information, which allows the quantitative estimation of the relationship between two variables, estimating a weighted effect size and increasing the precision of the estimators. In their process, they allow us to assess the quality of the methodology used, evaluate the consistency between the investigations, obtain a better global estimate and identify subgroups with different effects. This makes it possible to increase the validity of the conclusions of individual studies and identify areas of uncertainty where further investigation is necessary.

\section{Subjects, materials and methods}

The phases of a well-conducted meta-analysis are very similar to those of a primary investigation, with the exception that the observation unit is the studies instead of the patients. The first step is to define a good research protocol that clearly describes the reason for performing the meta-analysis, the objectives, and the hypothesis to be investigated. Then, a systematic and exhaustive search is performed for all potentially relevant articles, and then the selection of the articles to be included in the review using explicit inclusion and exclusion criteria. Finally, it will be necessary to summarize the data from the different articles in a well-structured way, which avoids errors and facilitates subsequent analysis. A description of the design of the original studies, the synthesis of the data obtained and the interpretation of the results will be made. All this scientific process must be reflected in the materials and methods section of the document to be published ${ }^{7}$.

In 1999 the QUORUM Declaration (Quality Of Reporting Of Meta-analysis $)^{8}$ was published with the aim of improving the quality of systematic reviews and meta-analyses. It offered the first norms for the publication of this kind of articles, and was updated until 2010, when the PRISMA Declaration (Preferred Reporting Items for Systematic reviews and Meta-Analysis $)^{9-10}$ was published. In this, unlike the previous one, each item that must be considered for the preparation of these studies is detailed and justified. To further develop a systematic review, it is suggested to carefully read these international standards and the Cochrane manual of systematic reviews ${ }^{7-9}$. All these articles are freely accessible on the Internet. 


\section{Analysis}

There are multiple limitations that must be considered when evaluating the results of a meta-analysis; for this reason, we will divide the analysis into four points, to discuss the problems and possible statistical solutions:

1. The quality of the studies included.

2. The publication bias itself.

3. The selection bias of the articles to be included in the meta-analysis.

4. The variability or heterogeneity in the estimation of the studies.

\section{The quality of the studies}

Consider the possibility of a series of clinical trials with a high methodological quality that examine the same intervention in a patient population. Each trial will provide an unbiased estimate of the effect of the same underlying treatment, the variability observed between trials can confidently be attributed to random variation, and the meta-analysis should provide an unbiased estimate of the treatment, with an increased precision of this estimate. A fundamentally different situation arises in the case of epidemiological studies, case-control studies, cross-sectional studies, and cohort studies. In these, due to confounding effects and biases, estimates may be generated that show associations that deviate from the effect in a systematic way, thus providing a falsely accurate estimate. In-depth examination of heterogeneity between the results of observational studies, in particular of possible sources of confusion and bias, will provide more ideas about the topic under review than the mechanical calculation of a weighted effect measure, for which a sensitivity analysis (that we will review later) can be used. The main difference between observational studies and randomized controlled clinical trials does not mean that the latter are immune to bias. Bias can also be introduced if the methodological quality of controlled clinical trials is insufficient. The quality of the studies can be determined using evidence-based medicine analysis techniques and published guidelines for such purposes ${ }^{1-4,7}$. Currently, the most frequently used guidelines to assess the quality of the evidence in articles are those of GRADE (Grading of Recommendations Assessment, Development and Evaluation https://www. gradeworkinggroup.org).

\section{Publication bias}

Meta-analyses have made possible to establish a publication bias when interpreting evidence. Publication bias refers to the fact that published scientific articles do not accurately reflect all the clinical trials conducted. Not including all studies is one of the main criticisms that meta-analyses have received. We can summarize such criticisms by noting that studies with statistically significant positive results are more likely to be sent for publication, accepted and published in journals with a higher impact than those that do not show differences; also, once accepted, the journal publishes them faster. They are also cited more frequently, which considerably increases the probability that they will appear in a database when conducting a literature search. Likewise, trials with a large number of patients are more likely to be published, even if their results are negative, this is, when there was no difference between the study group and the control group. On the other hand, many times, clinical trials that conclude a non-significant result or a result contrary to the hypothesis are not published, not only because the journals do not accept them, but also because the authors do not send them for publication, or because a great proportion of medical research is funded by pharmaceutical companies and they will inevitably be reluctant to publish papers when a new therapy does not show to be more effective than the currently approved one.

It can also happen that the same study has been published more than once, in an apparently different way. Duplicate publications can also introduce bias. There are articles that have been published up to 5 times with the same patients but with different titles. Some authors do this in order to increase their resume. This induces a bias in results by including more than once the records of the same article. There is no doubt, and there have even been studies that confirm it, that there is a bias in favor of certain types of publications, a significant bias in the selection of publication languages and a bias in the number of citations, which leads to a bias in the possibility that certain results are included or not in a meta-analysis. Of course, it must be clear that the bias is mainly related to the lack of articles with a small sample size with an effect that is less, null or opposite to the results of the analysis.

One method to assess this type of bias is to use the funnel plot proposed by Vandenbroucke in 1988 (Fig. 1). The funnel plot is based on the fact that the precision for estimating the effect of the underlying treatment will increase as the sample size of the studies increases: the 


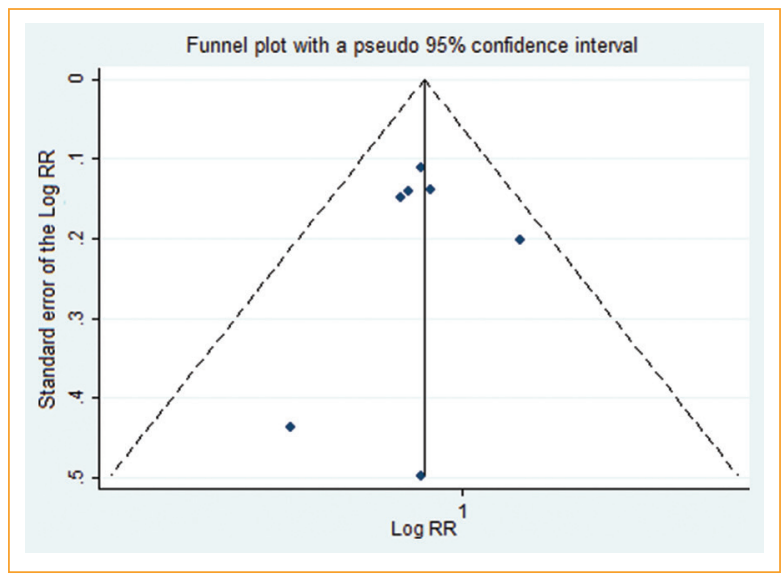

Figure 1. Funnel plot of the relative risk (RR) that evaluates the effect of an eye patch on the repair or healing of superficial corneal erosions of less than $10 \mathrm{~mm}^{2}$ on the first day. The plot appears to be symmetrical around the black line, which is the Log of the weighted RR of all studies. Some small studies around the Log RR between 0 and 0.5 might be missing. The segmented black lines of the edges correspond to a pseudo confidence interval $\pm 1.96^{*}$ standard error; the no difference is represented as the logarithm of the $R R=0$.

standard error of the natural logarithm of the measure of the effect of the relative risk (RR) or odds ratio (OR) (or the sample size) is recorded on the $Y$ axis, and the natural logarithm of the magnitude of the effect of the $\mathrm{RR}$ or $\mathrm{OR}$ is recorded on the $\mathrm{X}$ axis. The results of the small studies are widely dispersed as a cloud of points in the lower part of the plot around the central estimator, generating a progressive narrowing towards the vertex of the plot as the studies have larger sample sizes. In the absence of bias, the plot will resemble a symmetrical inverted funnel. If there is a publication bias of smaller studies, only those articles that show benefit of the treatment will be present, generating an asymmetric distribution of the points. Therefore, if there is bias, the funnel plot will often be uneven and asymmetric.

A simple funnel plot analysis is a useful test for the potential presence of publication bias in a meta-analysis, but this ability to detect bias will be limited when the meta-analyses are based on a small number of clinical trials, as the points cloud will be small; therefore, the results should be analyzed with caution. What's more, even if there is a publication bias, it may not result in an asymmetric funnel plot, for example, when the plot of the points cloud is hollowed out.

A funnel plot is not a very reliable method for evaluating publication bias, although it does give us an idea of whether the study results are spread symmetrically

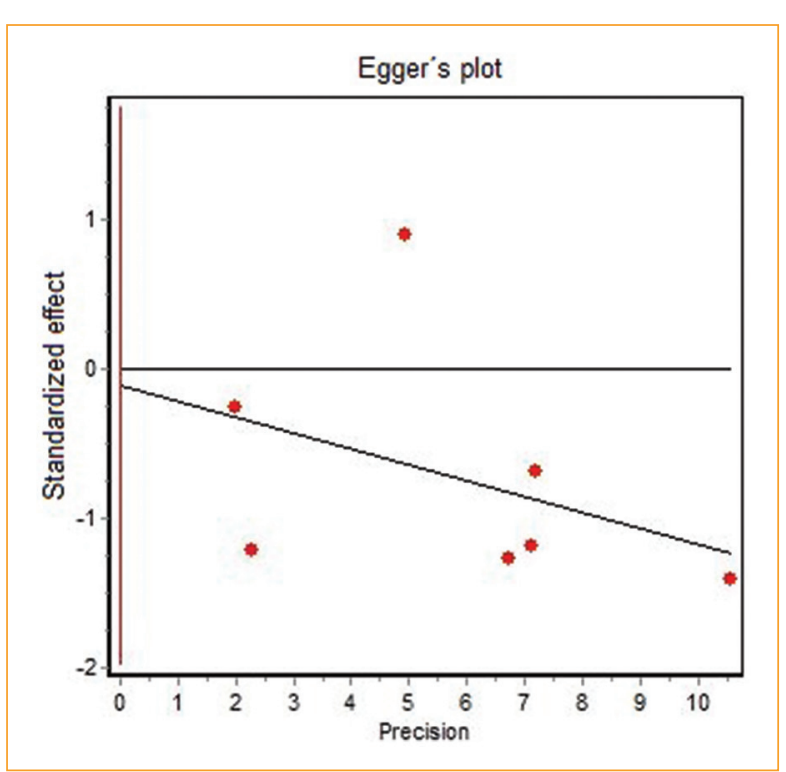

Figure 2. It shows the dispersion of points of the different studies according to their precision. The black line corresponds to the regression of Egger's test, which reaches 0 , so there is no evidence of bias from small studies.

around a more precise central effect. An asymmetric funnel plot may be due to publication bias, but it may also result from clinical heterogeneity between studies (for example, different event rates) or methodological heterogeneity between studies (for example, failure to blind allocation); therefore, rather than publication bias, this tends to be considered as if the result of the meta-analysis is influenced or not by the effect of studies with a small sample size.

Symmetry or asymmetry has generally been defined informally, through visual examination of these charts. As expected, funnel plots have been interpreted differently by different observers; therefore, funnel plot asymmetry has been measured numerically by a simple asymmetry test, predicting discordance of results when meta-analyses are compared to large clinical trials. There are some statistical tests used to detect the asymmetry of the funnel; therefore, it is advisable to always complement the study of graphical bias with more objective statistical tests, such as the Egger test (1997) and the Begg and Mazumdar test (1994). These tests contrast the null hypothesis of absence of publication bias (Fig. 2). The Begg and Mazumdar test evaluates if there is a correlation between the standardized magnitude of the effect and its variability. If for a greater standardized effect there is a greater variance, it means that the small studies (of large 
variance) are published or were selected when the effect is different from the effect of large studies (of small variance). The correlation is calculated with Kendall's tau ordinal correlation coefficient, and the absence of statistical significance suggests that there is no publication bias. The Egger $^{13}$ test is more specific than the Begg's test and can yield false positives. The method consists on fitting a regression line to the points with an abscissa equal to precision, which is the inverse of the standard error 1/ES (independent variable), and an ordinate equal to the standardized effect "In RR/ES" (dependent variable). If there is no publication bias, the ordinate at the origin will be equal to zero, and the further from zero it is, the more pronounced the asymmetry and the greater the evidence of bias (Fig. 2). When the Begg and Mazumdar test, which is a rank correlation test, does not yield a significant result $(p>0.10)$, caution is advised when interpreting it, since it has very little power when the number of studies is small, as it frequently occurs.

Macaskill, et al. (2001) proposed another measure of asymmetry. This is calculated by fitting a linear regression model between the weighted effect (Ln OR or RR) and the sample size. In 2006, Peters, et al. found that this method works best if the independent variable is the reciprocal of the sample size $(1 / n)$. The interpretation is the same as with the other tests, but this method is less sensitive than Egger's for detecting publication bias, although it is more specific. The Begg and Mazumdar method is an intermediate between the two.

Another tool to assess the risk of bias is the Trimand-Fill, a method developed by Duval and Tweedie (2000). It is a non-parametric method for quantifying the risk of publication bias in a meta-analysis. The method estimates the number and results of studies that might be missing and adjusts the meta-analysis to incorporate missing imputed data. The authors state that this method is effective and consistent with other adjustment techniques. This provides a funnel plot with the data marked, in this case with a square, unlike the circle of the articles or with the same symbol, but with a different color. It also allows the calculation of the global weighted measure (OR or RR) for the fixed or random effects model, with and without studies calculated as missing to assess how much the results change.

In the previous example in the plot of Figure 1, Beg$g$ 's test was $p=0.7639$ and Egger's test was $p=0.8785$. No studies were deemed to be missing in this analysis using the Trim-and-Fill method.

\section{Choice or selection bias}

Selection bias is another of the limitations of a meta-analysis, so it is very important to clearly define the inclusion and exclusion criteria of the articles, being as objective as possible. Selection bias will arise when only some of the research articles conducted are included in the meta-analysis, as a consequence of not performing an exhaustive literature search or having tendentiously limited it or having improperly eliminated articles due to lack of validity. The author of the meta-analysis is the one who biases the results, since his/ her criteria for including or excluding a study from the analysis may be influenced. Both in the incorporation and in the exclusion of articles it is necessary to be very careful; the ideal scenario is that two independent evaluators decide and, in case of a discrepancy, a third evaluator makes a final decision. This type of bias can only be handled by the study design and not by the statistics of the data analysis. Guidelines such as PRISMA, STROBE, CONCORT or the Cochrane Handbook for Systematic Reviews of Interventions can help minimize these methodological biases s $^{1-4,7-10}$.

\section{Estimation of the effect and homogeneity analysis}

As one of the objectives of the meta-analysis is to group the information provided by different studies, a crucial issue is to determine a standardized measure of the effect that allows precisely this combination. Before conducting the meta-analysis, the researcher has to establish the measures to be used to describe and represent the effects, and thus be able to incorporate them. Obviously, these measures will depend on both the type of response (binary or continuous) and the study design (experimental or observational) used in the primary studies:

- Binary response: this type of response only has two outcome categories (death or survival, sick or healthy, success or failure, vision improvement or lack of vision improvement, controlled or uncontrolled intraocular pressure, glaucoma progression or no progression, etc.). In studies with this type of response, the most commonly used measures of effect are the hazard ratio (relative risk) and the odds ratio.

- Continuous response: in the medical literature there are many studies in which the response variable is measured on a continuous scale (intraocular pressure, endothelial cell count, retinal thickness by optical coherence tomography, pachymetry, etc.). If the 
Table 1. Summary of the main statistical methods used according to type of data and model

\begin{tabular}{|l|l|l|l|}
\hline Type of variable & Statistical measure & Model & Methods \\
\hline Type of data & Odds ratio (OR) & Fixed effects & Peto \\
\hline Dichotomic & & Mantel-Haenszel \\
\hline & Relative risk (RR) & Random effects & DerSimonian and Laird \\
\hline Continuous & Fixed effects & Mantel-Haenszel \\
\hline Risk difference & & Wolf \\
\hline & Random effects & DerSimonian and Laird \\
\hline Mean difference & Fixed effects & Mantel-Haenszel \\
\hline & Random effects & Wolf \\
\hline Standardized mean difference & FerSimonian and Laird \\
\hline & Fixed effects & Wolf \\
\hline
\end{tabular}

responses from all the studies are measured on the same scale, then the effect measure or effect size will be just the difference between the group means. If this was not the case, that is, if the studies measure the response on a different scale, a standardized difference of means should be considered instead (Table 1).

To present this weighted effect measure, a plot is constructed, known as a forest plot, in which, on the abscissa ( $X$ axis) the effect measure (OR or RR) is represented, and along the ordinate axis ( $\mathrm{Y}$ axis) the different studies are located, generally ordered by the year of publication or by any other criterion (Fig. 3). For each study and for the global estimation of the effect, its point estimate and also the confidence interval $(\mathrm{Cl})$, usually $95 \%$, are represented, assuming a fixed-effects and/or a random-effects model. In Figure 3, zone C shows the graphic part and zone $D$ shows the numerical values. A vertical line is also drawn, located at the point where there is no difference between the events studied, corresponding to the continuous black line drawn at $R R=1$, and the red segmented line corresponds to the weighted value of the RR. On the sides of the study, the name of the first author and the year of the study are annotated, and sometimes the number of existing events for each situation studied (zones $A$ and $\mathrm{B}$ ) is identified.
The problem with this type of plot is that the studies with the worst precision, those with the least weight in the estimation of the global effect, are those with the greatest visual impact, since their $\mathrm{Cl}$ is the widest. To remedy this, in some way, the value of the mean effect is represented with a symbol whose area is proportional to the precision, in such a way that the largest and, therefore, most striking symbols correspond to the most precise studies with the greater weight in the estimation of the weighted effect (gray square on the study in zone C). Finally, the value of how much each study weighs is placed in the weighted estimate (zone E). In the lower part of the plot, the weighted effect measure is placed, in this case the RR with its $95 \% \mathrm{Cl}$, and it is plotted using a horizontal diamond, where the extremes correspond to the $95 \% \mathrm{Cl}$. Also, the homogeneity statistical tests (that will be mentioned later) are annotated.

It is crucial to understand the limitations of meta-analyses and the importance of exploring sources of heterogeneity and bias to ensure that the conclusions are valid. When we speak of heterogeneity we can distinguish two aspects. On one hand, the differences between the studies in terms of the characteristics of the patients included, the methodology used, the follow-up time, the doses used, the geographical location, etc., and on the other hand, the concept of statistical 


\section{Healing on the first day of treatment}

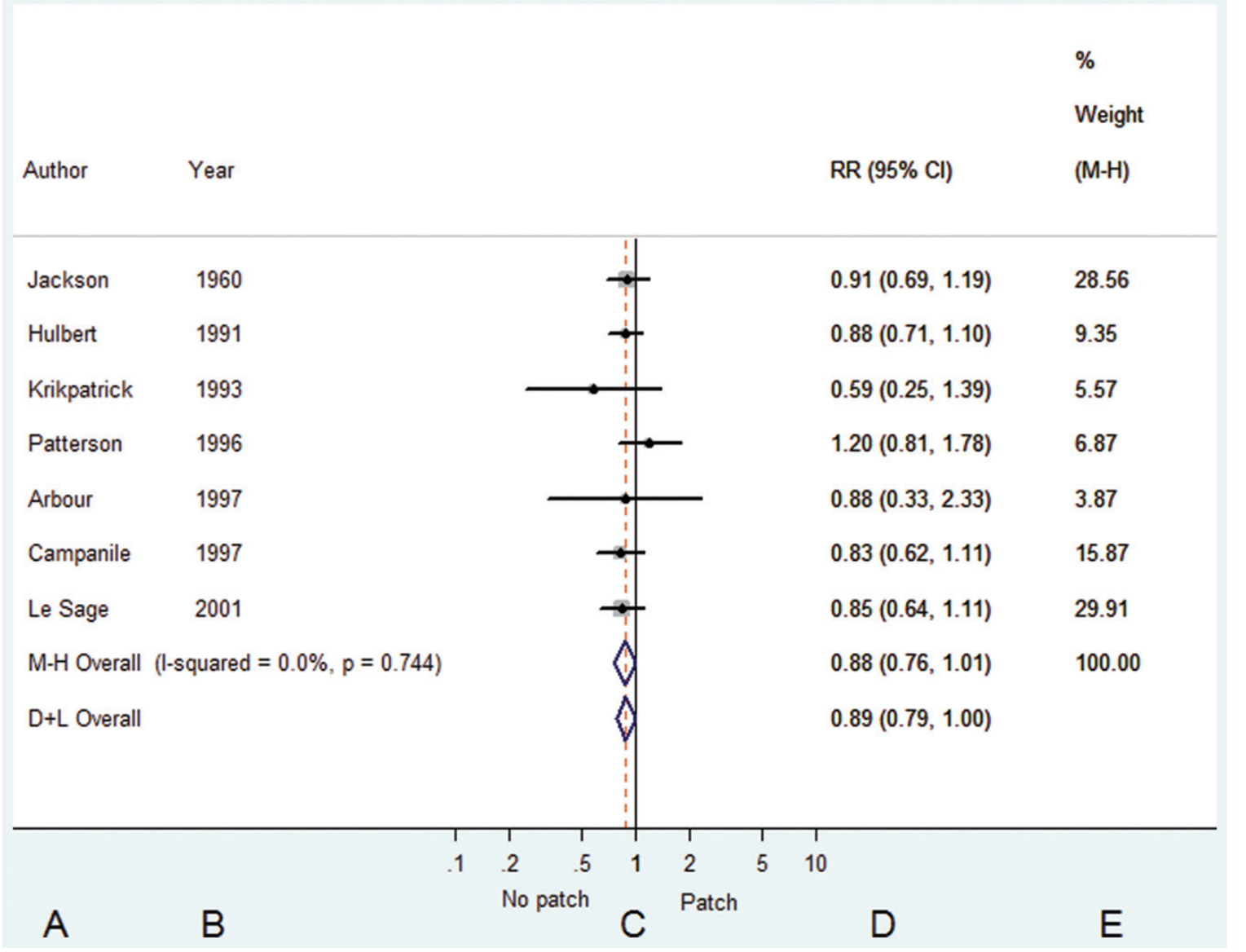

Figure 3. It can be seen how the value of the relative risk (RR) changes in the lower zone from 0.88 in fixed effects (Mantel-Haenszel [M-H]) to 0.89 in random effects (DerSimonian and Laird [D+L]). The estimate becomes more conservative, then, next to each estimate, the $95 \%$ confidence interval $(\mathrm{Cl})$ varies, the random effects interval is broader and therefore, less precise than the fixed effects interval. In parentheses, next to $\mathrm{M}-\mathrm{H}$, the estimation of $1^{2}=0.0 \%$ and $\mathrm{p}=0.744$ of the $\mathrm{X}^{2}$ of the $\mathrm{M}-\mathrm{H}$ analysis is presented for this case, which establishes that there is no statistical heterogeneity.

heterogeneity, which only quantifies the variability between the results of the studies, and that may be due to real differences in design and conduction between the included studies or other causes. One of the aspects of heterogeneity, related to clinical homogeneity, is a methodological problem, since it will be necessary to decide whether the differences between the studies, which always exist, allow or not to combine them, regardless of the results obtained and, therefore, this is prior to the conduction of the meta-analysis ${ }^{2,3}$. Studies must be compatible in order to be combined and must have at least three similar aspects: the treatments used, the patients included, and the outcome variables used. Statistical heterogeneity aims to quantify the variability of the measured result regarding the result of the weighted effect, and to determine if such variability is greater than what would be expected by pure chance. The ideal situation for the application of a meta-analysis in terms of determining a weighted effect occurs when the studies that are combined are randomized clinical trials and their results are homogeneous, even though the size of the original sample was insufficient to obtain conclusive results. When there are discrepant studies, the investigation of the presence and causes of heterogeneity in the results may become the main objective of the meta-analysis, and displace the objective of obtaining a weighted effect.

Two statistical models have been developed to make inferences about the weighted effect of a group of studies: the fixed effects model and the random effects 
model. The fixed effects model is known as the inverse variance-weighted method, and it was described in 1930 by Birge and also by Cochran. It assumes that the effect size is a fixed and unknown constant that we must estimate, that the individual studies are homogeneous and come from the same population, and that the differences between the effect size estimates are only due to variability within studies (sampling error); it is usually calculated with the Mantel-Haenszel (MH) method. The random effects model considers that the size of the effect comes from a distribution of parameters with a mean and from inter-study variance. In this case, the differences in the effect size estimates are due to intra-study variability and inter-study variability, which can be estimated by different methods, the most frequentbeing the DerSimonian-Laird $(D+L)$ and maximum likelihood. The differences between both methods are minimal, since the first is based on the method of moments and the second on the maximum likelihood function; both methods usually give very precise and similar estimators, the first being the most used (Table 1).

The random effects model, by considering variability not only within studies but also between studies, allows to make an inference that goes beyond the group of studies with which we are working. In contrast, the fixed effects model only allows to reach inferences that are conditioned to the sample of studies.

The random effects model does not assume a single parametric effect size, but rather a normal distribution of effect size parameters, thus including an inter-study variance, in addition to the intra-study variance, for the effect size estimates for each study. The parametric variance of the random effects $\left(T^{2}\right)$ is a measure of inter-study heterogeneity. From this point of view, the fixed effects model is a special case of the random effects model, where $T^{2}=0$. For the random effects model, the mean effect size is also a measure weighted by the inverse of the variance, but in this case the variance has two components, within-subject and inter-subject variance. This means that, in the presence of heterogeneity, the random effects model should be used. The random effects model, by considering more variability, produces larger $\mathrm{Cls}$ than the fixed effects model and, in addition, increases the importance of small studies, in which publication bias is more frequent, as opposed to fixed effects, where studies with a larger sample size will have more weight.

Random effects models should be used whenever it is determined, after an analysis of heterogeneity ${ }^{14,15}$, that the studies are not homogeneous and the causes cannot be clearly identified, which is usually common, especially in epidemiological studies. A statistic defined by Cochran in 1954 is usually used to evaluate statistical heterogeneity. It is based on calculating the sum of the squared deviations between the individual result of each study and the global result, weighted by the same weight with which each result intervenes in the global estimation: $Q w=\Sigma w_{i}\left(d_{i}-d\right)^{2}$. The null hypothesis of homogeneity is $H_{0}: T^{2}=0$. If the $Q_{w}$ test yields a significant value, we reject the null hypothesis of homogeneity between the studies and apply the random effects model. To see if $Q_{w}$ is significant, it is contrasted against a $X^{2}$ with $n-1$ degrees of freedom. The problem is that the $Q_{w}$ test has a low power, so in many cases we are assuming homogeneity and applying fixed effects when in fact we should apply random effects. If the number of studies is small, their ability to detect heterogeneity is very low (low contrast power), whereas, on the contrary, when the meta-analysis combines a large number of studies, the result can be statistically significant, even when the magnitude of heterogeneity is not of clinical relevance. This is nothing new, these are the problems inherent to the methodology of statistical contrast tests. Since the most common situation in meta-analyses is a small number of studies, it is recommended to use a rejection level in this test of at least $p>0.1$, instead of the traditional $p>0.05$.

The limitations of the $Q_{w}$ test mentioned above, as well as the fact that its result does not serve to compare different meta-analyses in which different numbers of studies are involved, have led to the search for other indexes that allow the quantification of heterogeneity so that the estimated parameter serves to compare different meta-analyses, and thus, in some way, weigh the validity of the mean effect calculated. In this regard, an index called $l^{2}$ has been proposed, based on the idea that the question of interest is not whether or not there is statistically significant heterogeneity, but how it affects the conclusions of the meta-analysis. The authors tried to find an index that allows comparison between different meta-analyses and, therefore, does not depend on the unit of measurement used to quantify the effect, or on the number of included studies, and that is also easily interpretable by people who are not experts in statistics. The parameter $\mathrm{I}^{2}$ indicates the proportion of the variation between studies regarding the total variation, that is, the proportion of the total variation that is attributable to heterogeneity, and is expressed in percentages (\%). The authors who developed it propose values of 25,50 and $75 \%$ as possible thresholds to indicate low, moderate or high heterogeneity, respectively. Once again, it should be noted that 
the absence of statistical heterogeneity does not guarantee that the studies are homogeneous in terms of their clinical, biological or methodological characteristics, and therefore, does not confirm the validity of combining them (Fig. 3).

Due to the low power of the existing statistical tests, these can be complemented with some graphical representations that allow visual inspection of the magnitude of the variability between studies. The most used representations are the Galbraith chart (Fig. 4), recommended for any type of study (observational and experimental), and the L'Abbé plot (Fig. 5), more restrictive, applicable only to meta-analysis of clinical trials. The Galbraith plot represents the precision of each study (the inverse of the standard error) versus the standardized effect; likewise, it represents the regression line adjusted to these points and a confidence band. Studies that fall outside this band are the ones that contribute the most to heterogeneity. On the other hand, the position of the studies on the $X$ axis makes it possible to visually identify those that have a greater weight in the meta-analysis. This plotcan also be used to detect sources of heterogeneity by labeling studies with different variables, such as the year of publication.

Another approach to determine the presence of heterogeneity between studies is to identify the possible cause. A natural question in this context is whether there is a relationship between the response rate and the baseline risk. A good graphical tool to verify this is the socalled L'Abbé plot (Fig. 5), in which for each study, the frequency of events in the treatment group is represented on the $\mathrm{Y}$-axis versus the frequency of events in the control group, on the $\mathrm{X}$-axis. This plot only occurs when the response variable is binary. A line is drawn at $45^{\circ}$ that divides the plot in two sections. On one side of this line we will have the studies that were favorable to the treatment group and on the other side the studies that were favorable to the control group; in addition, a segmented line is drawn with the value of the weighted effect measure, in this case the RR $=0.88$. Points of different sizes are usually represented with an area inversely proportional to the variance of the effect estimation in each study (directly proportional to the precision of the estimate), in such a way that, the larger a point, the greater the precision of the study it represents. The presence of scattered points in the L'Abbé plot indicates heterogeneity, while, if they are grouped in a narrow area, it is a sign of more homogeneous results. The presence of patterns in this graph can suggest what is happening. If the effectiveness depends on baseline risk, it could be that, for low baseline

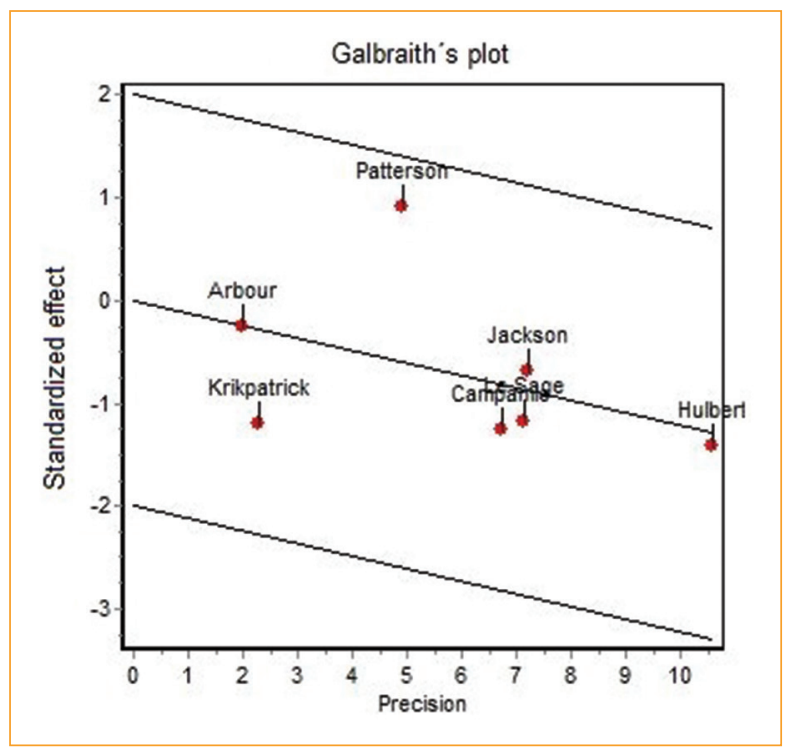

Figure 4. The Galbraith plot represents the precision of each study against the standardized effect. It can be seen how, in this case, all the studies are between the confidence bands and most of the studies are around the adjusted regression line; therefore, there is not much heterogeneity between the studies.

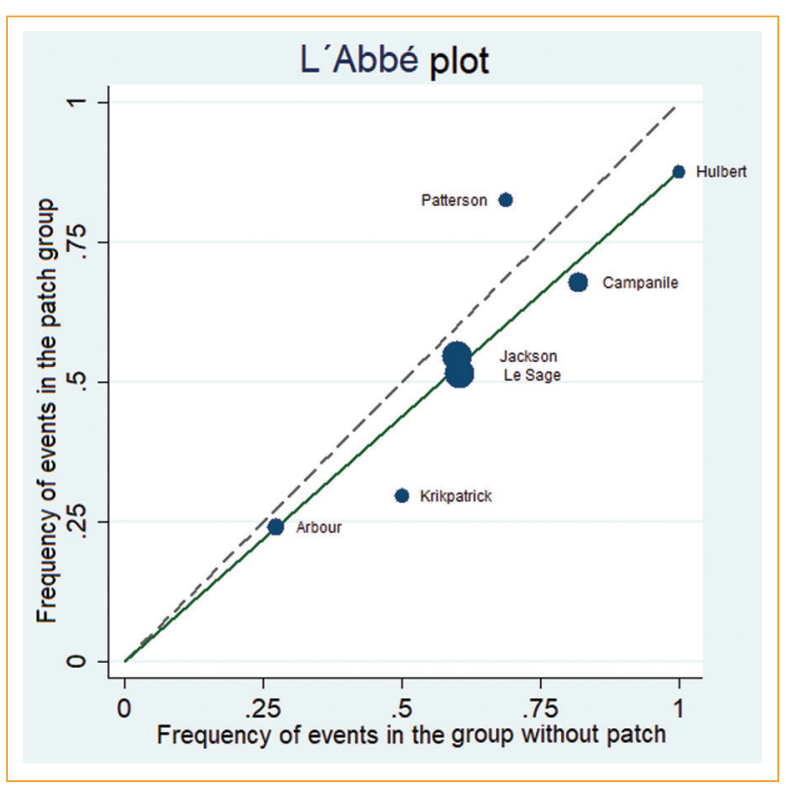

Figure 5. The L'Abbé plot allows to evaluate the studies that favor one or another treatment regarding the rate of events in each group, visually and in a very easy way.

risks, the points are very close to the dividing line or even on the side of ineffectiveness, and as we move towards higher baseline risks, the points move away from that line towards higher efficacy values. 
We can further the idea that some underlying characteristic of the studies may be affecting the heterogeneity of the results, not only the baseline risk, but any other characteristic. This can be used to explore sources of heterogeneity and test new hypotheses that were not raised in individual studies, by using meta-regression techniques. The term meta-regression describes the analysis in which the characteristics of the studies or of the subjects included in each study are used as explanatory variables in a multivariate regression mod$\mathrm{el}$, in which the dependent variable is the measured effect or some measure of the deviation of the effect of each study regarding the weighted effect. Here, the unit of observation is each study or each subgroup of studies, and when estimating the coefficients of the regression equation, methods are used that weight the intervention of each study in that estimate, according to its precision (proportional to the study size, inversely proportional to the variability of the study). Since the sample is small (number of studies), the number of independent variables involved in the regression equation must be small. As in traditional regression analysis, the rule of 5 or 10 cases for each variable is used, and since the number of studies in a meta-analysis is usually small, not many variables should be used in the regression equation. A typical example of meta-regression, now classic, is the one that presents the "In odds ratio" of the efficacy of the BCG (Bacillus Calmette-Guerin) vaccine against tuberculosis compared to the latitude of the country in which the study was conducted. In the study performed, it was observed that the risk of vaccine ineffectiveness probably tends to increase when we approach the planet's equator.

Finally, if it is suspected that there are reasons that may explain the heterogeneity of the results between studies, the most advisable option is to perform a subgroup analysis that only combines the studies that meet a certain condition or characteristic, so that these are more homogeneous. The idea of subgroup analysis or stratification consists in performing the meta-analysis in different groups of studies, combined according to their characteristics (published studies versus unpublished studies, large versus small studies, studies according to quality level, publication date, etc.) or per the characteristics of the patients included, and verify how the result varies by estimating it again each time.

The sensitivity analysis aims to evaluate the influence of each of the studies on the estimation of the weighted effect, and, therefore, the robustness or stability of the final measure obtained. This analysis consists on repeating the meta-analysis as many times as the number of studies, so that each time the weighted effect is calculated, one study is omitted and the rest are used. If the results of the different meta-analyses are similar, and, therefore, the effect has the same direction, magnitude and statistical significance, it can be concluded that the results are robust. Otherwise, there would not be a robust estimator, which would require some caution in the interpretation of the results or could be a reason to generate new hypotheses. An appreciation to keep in mind is that, when accurate studies with a good quality are withdrawn, the magnitude of the final result is expanded, which allows identifying these types of studies. It can be represented graphically by a forest plot, called an influence plot, in which the weighted effect is plotted for each analysis, always excluding an article. The sensitivity analysis can also be used to evaluate the influence on the results of the meta-analysis of certain aspects related to the validity of the studies, such as, for example, the exclusion of studies that do not exceed a certain quality threshold; unpublished works; or studies in which there is uncertainty about the fulfillment of the selection criteria. Furthermore, it is then possible to estimate the number of studies with zero effect, conducted and not published, that should exist to modify the meaning of a possible "positive" conclusion obtained with a meta-analysis. This is called "Fail-Safe N", and the methods of Rosenthal (1979) and Orwin (1983) are used. If this number is very high, the probability that publication bias has substantially modified the results is considered low, and the existence of the differences suggested by the meta-analysis is accepted.

Another important analysis method is the cumulative meta-analysis. This is defined as the process in which a new meta-analysis is carried out each time a new published study appears (Fig. 6). This type of meta-analysis does not require special statistical techniques to combine the studies. The statistical combination of the results of the studies will be performed as many times as there are studies, minus one. So initially, the first two are combined and, in successive meta-analyses, each new study is incorporated one by one. Although in the accumulated meta-analysis the chronological order is usually the most frequently used (year of publication), sometimes it is interesting to perform other types of study aggregation in an independent or complementary way, based, for example, on the increasing order by sample sizes; differences between treatment and control; study quality scores, etc. A cumulative meta-analysis also makes it possible to retrospectively study the moment or year of publication in 


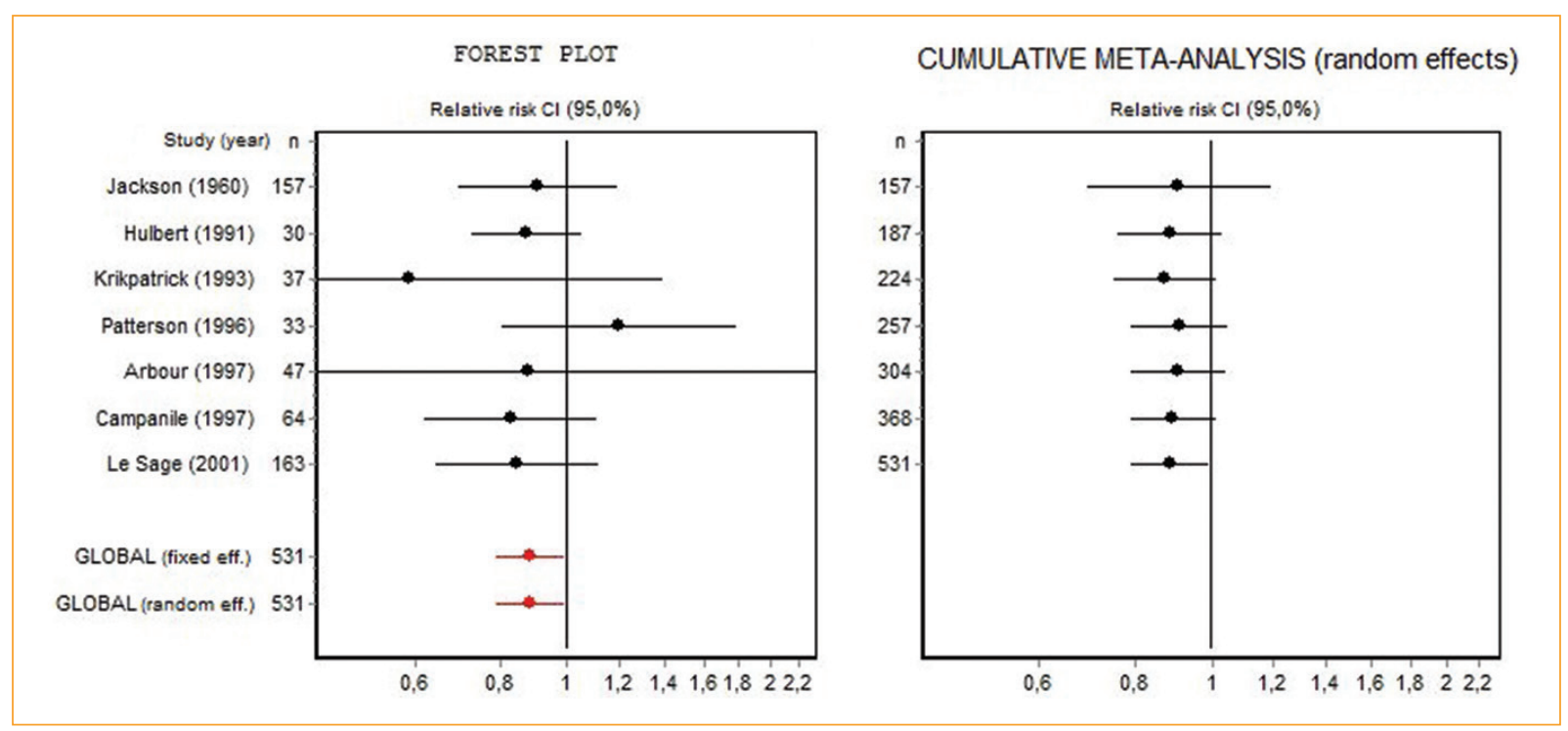

Figure 6. On the left is the normal meta-analysis ordered by year of publication. To the right is a cumulative analysis, where it can be observed how the estimate and the $95 \%$ confidence interval (CI) changes as different studies are added, and how in recent years there are almost no changes in these values despite adding new studies.

which the effect of the treatment first reached the conventional levels of statistical significance, when a new treatment exceeds the control. This form of presentation of results highlights how difficult it is for any individual study, once relatively stable results have been achieved, to provide additional information. In fact, a study has to be large enough and deliver extreme enough results to substantially modify the cumulative results.

Finally, we will comment on the discrepancies that arise in the literature between large clinical trials and meta-analyses. LeLorier, et al. reviewed the discrepancies between 12 large clinical trials of more than 1,000 patients and 19 meta-analyses that attempted to answer the same research questions ${ }^{16}$. The degree of agreement was moderate between both types of investigations, the meta-analyses presented positive predictive values of $68 \%$ and negative predictive values of $67 \%$, with a sensitivity of $65 \%$ and a specificity of $70 \%$. Although part of the discrepancies observed seem to be due to an inadequate consideration of the heterogeneity of the original studies in the meta-analyses, large clinical trials and meta-analyses play complementary roles in the evaluation of clinical evidence, differentiated by the heterogeneity of the patients involved, whether these are mega-clinical trials or meta-analyses of smaller clinical trials.

\section{Final analysis of the example}

Regarding the examples used in the article of the data extracted from the Cochrane review that evaluates the use of an eye patch, we are going to summarize the full analysis sequentially. First, the variable used is dichotomous, whether or not the cornea is repaired on the first day after the eye is patched or not, with a corneal damage of less than $10 \mathrm{~mm}^{2}$. This, according to Table 1, forces us to decide to use the RR or OR through a fixed or random effects model. In this case, the RR was used by means of a fixed effects model with the $\mathrm{M}-\mathrm{H}$ method. This is the product of the type of studies (controlled clinical trial) plus the fact that in the analysis of heterogeneity (lower left part of Figure 3), both the $1^{2}=0.0 \%$ and the $p=0.744$ of the $X^{2}$ of the analysis of heterogeneity using $\mathrm{M}-\mathrm{H}$ showed very low heterogeneity between articles and no significant differences, respectively. From this it is concluded that the analyzed data can be meta-analyzed, but are all the articles included or the data are biased? To answer this we go to Figure 1. In the funnel plot, the articles are distributed more or less homogeneously except in the lower right part, where some articles might be missing, so we made an Egger's plot (Fig. 2), where the regression tends to 0 , and we also estimated the $p$-value of Egger's test, which was not significant ( $p=0.8785)$; therefore, the results do not seem to be affected by small studies or publication bias. To further confirm this, we used the Trim-and-Fill method, which showed that there were no missing studies in the analysis. If we combine all of the above with a suitable search and article selection protocol, then we can rest 
assured that the results can be correctly interpreted. Finally, the results are taken from Figure 3 , which are a $\mathrm{RR}=0.88$ with a $95 \% \mathrm{Cl}$ of 0.76 to 1.01 . This, together with the trend shown by the accumulated meta-analysis in Figure 6, helps to conclude that the use of an eye patch in patients with superficial corneal erosions of less than $10 \mathrm{~mm}^{2}$ does not report any benefit regarding the repair rate on the first day. Moreover, in the best scenario, according to the 95\% $\mathrm{Cl}$, the repair rate would be equal to not patching; therefore, the medical conduct according to this analysis would be not to patch these types of patients, since it is It is highly unlikely that a new clinical trial will have such resounding results in favor of the patch that when incorporated into the analysis it not only deviates the RR above 1, but also completely deviates the $95 \% \mathrm{Cl}$ above 1 , which would be the the only way that it could lead to a behavior change.

\section{Programs}

There are many programs that can be used to perform a meta-analysis, the vast majority of them are used to perform a frequentist analysis. Among the two best free programs are the RevMan (Review Manager) developed by the Cochrane Collaboration, which also helps to manage the manuscript and the study references, and the Metafor package of the $\mathrm{R}$ program. Within marketed programs that require payment, one of the best is Stata, since it has excellent graphics and allows updating with the different tests that are developed over the years; the examples were developed mainly with this program.

\section{Conflicts of interest}

The authors declare no conflicts of interest.

\section{Ethical disclosures}

Protection of human and animal subjects. The authors declare that no experiments were performed on humans or animals for this study.

Confidentiality of data. The authors declare that no patient data appear in this article.

Right to privacy and informed consent. The authors declare that no patient data appear in this article.

\section{References}

1. Oxman A, Cook D, Guyatt G. The Evidence Based Medicine Working Group. JAMA. 1994;272:1367-71.

2. Davidoff F, Haynes B, Sackett D, Smith R. Evidence based medicine BMJ. 1995:310:1085-6.

3. Sackett D, Straus S, Richardson W, Rosenberg W, Haynes R. Evidence - Based Medicine. How to Practice and Teach EBM. $2^{\text {nd }}$ ed. Edinburgh: Churchill Livingstone, 2000.

4. Guyatt G, Rennie D. Users' Guides to the Medical Literature. A Manual for Evidence-Based Clinical Practice. Chicago: AMA Press, 2002.

5. Egger M, Smith G, Altman D. Systematic Reviews in Health Care. Meta-analysis in context. London: BMJ; 2001.

6. Smith M, Glass G. Meta-analysis of psychotherapy outcome studies. American Psychologist. 1977;32:752-60.

7. Centro Cochrane Iberoamericano, traductores. Manual Cochrane de Revisiones Sistemáticas de Intervenciones, versión 5.1.0 [Internet]. BarceIona: Centro Cochrane Iberoamericano; 2012. Disponible en: https://es. cochrane.org/sites/es.cochrane.org/files/public/uploads/manual_cochrane 510 web.pdf

8. Moher D, Cook D, Eastwood S, Olkin I, Rennie D, Stroup D; for the QUOROM group. Improving the quality of reporting of meta-analysis of randomized controlled trials: The QUOROM statement. Lancet. 1999;354:1896-900.

9. Liberati A, Altman D, Tetzlaff J, Mulrow C, Gotzsche P, loannidis J, et al., and the PRISMA Group. The PRISMA statement for reporting systematic reviews and meta-analyses of studies that evaluate health care interventions: Explanation and elaboration. PLoSMed. 2009;6: e1000100. Epub 2009 Jul 21.

10. Urrutia G, Bonfill X. Declaración PRISMA: una propuesta para mejorar la publicación de revisiones sistemáticas y metaanálisis. Med Clin (Barc). 2010;135: 507-11.

11. Fau C, Nabzo S, Nasabun V. Metaanálisis en red. Rev Mex Oftalmol. 2018;92(3):153-9. Disponible en: https://www.rmo.com.mx/frame_esp.php?id=47.

12. Turner A, Rabiu M. Patching for corneal abrasion. Cochrane Database of Systematic Reviews 2006, Issue 2. Art. No.:CD004764. DOI: 10.1002/14651858.CD004764.pub2

13. Egger M, Davey S, Schneider M, Minder C. Bias in meta-analysis detected by a simple, graphical test. BMJ. 1997;315(7109):629-34.

14. Higgins J, Thompson S. Quantifying heterogeneity in a meta-analysis. Stat Med. 2002; 21(11):1539-58.

15. Higgins J, Thompson S, Deeks J, Altman D. Measuring inconsistency in meta-analyses. BMJ. 2003;327:557-60.

16. LeLorier J, Grégoire G, Benhaddad A, Lapierre J, Derderian F. Discrepancies between meta-analyses and subsequent large randomized, controlled trials. N Engl J Med. 1997;337(8):536-42. 